\title{
及2-adrenergic agonists do not improve physical performance in healthy individuals
}

To the Editor,

All beta-2 agonists are prohibited by the World Anti-Doping Agency (WADA, www.wada-ama.org). However, its use may be permitted in athletes who are granted a therapeutic use exemption (TUE).

As the evidence remains controversial, there has been increasing debate on whether $\beta 2$-agonists have the potential to improve physical performance. ${ }^{1}$
Therefore, this study aimed to systematically review the available scientific evidence on the effect of $\beta 2$-agonists on physical performance in healthy, nonasthmatic individuals.

All randomized controlled studies, written in any language, were considered. A fixed or, when necessary, a random-effects meta-analysis was performed for two outcomes $\left(\mathrm{VO}_{2 \max }\right.$ and endurance time to exhaustion (TTE)), considering the study design, substance,

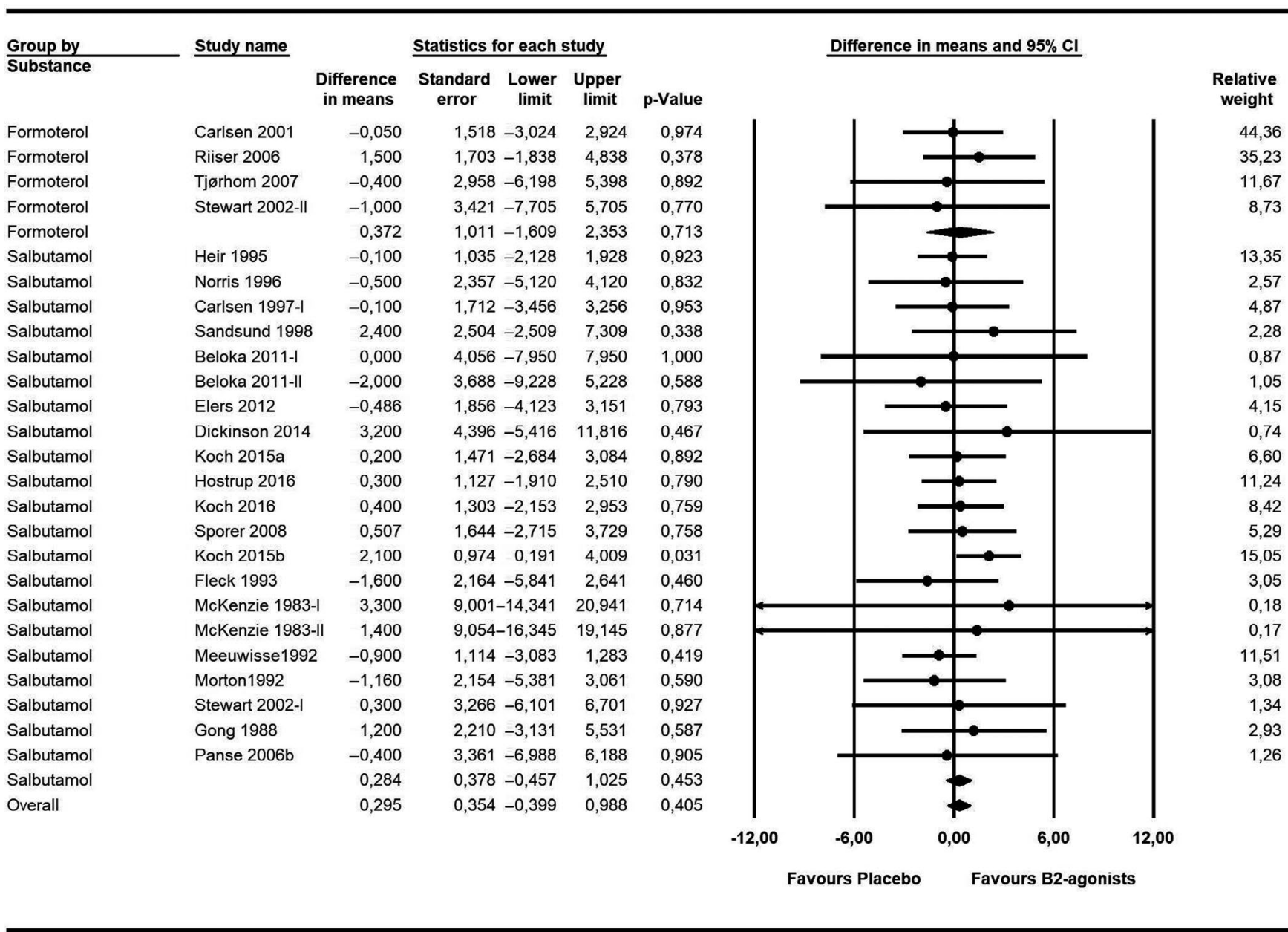

FIGURE 1 Forest plot representing the comparison of $\mathrm{MD}$ of $\mathrm{VO}_{2 \max }$ between salbutamol, formoterol and placebo. The letters "a" and "b" following the author's name correspond to different studies by the same author published in the same year. The Roman numbering presented after the author's name corresponds to the same study, but different interventions (more than one $\beta 2$-agonist or more than one dose) 
administration and gender of the participants. The mean difference (MD) was used as an effect size measure. Publication bias was assessed using the funnel plot, Duval and Tweedie's trim and fill method, and Eger's regression test (See supporting information for details). The selection process of the studies included in the systematic review and meta-analysis is presented in Figure $\mathrm{S} 1$ of the supporting information.

The fixed-effects meta-analysis studying the effect of $\beta 2$-agonists on $\mathrm{VO}_{2 \max }$ showed no significant effects of salbutamol vs placebo $(M D=0.284 ; P$-value $=.45)$, of formoterol vs. placebo $(M D=0.372 ; P$-value $=.71)$, and neither of overall $\beta 2$-agonists vs. placebo (MD $=0.295$; $P$-value $=.40$ ) (Figure 1$)$. (For summary of meta-analysis, sensitivity analysis and evaluation of publication bias, see supporting information).

Two fixed-effects meta-analyses were performed considering the different routes of administration of salbutamol. In the meta-analysis considering the effect of inhaled salbutamol on $\mathrm{VO}_{2 \max }$, no statistically significant effect was shown: $M D=0.323 ; 95 \% \mathrm{Cl}$ : $(-0.47857 ; 1.123) ; P$-value $=.43$. Similar results were obtained in the meta-analysis considering the effect of systemic salbutamol on $\mathrm{VO}_{2 \text { max }}: \mathrm{MD}=0.053 ; 95 \% \mathrm{Cl}$ : $(-1.898 ; 2.003) ; P$-value $=.95$. (For summary of meta-analysis, sensitivity analysis and evaluation of publication bias, see supporting information).

To assess the effect of $\beta 2$-agonists on TTE, three meta-analyses (fixed-effects, random-effects and mixed-effects) were performed, considering different percentages of $\mathrm{VO}_{2 \text { max }}: \geq 100 \% \mathrm{VO}_{2 \max },<100 \%$
$\mathrm{VO}_{2 \max }$ and mixed-effects. In the fixed-effects meta-analysis that considered endurance TTE $\geq 100 \%$ of $\mathrm{VO}_{2 \max }$, no statistically significant difference was found between $\beta 2$-agonists and placebo $(M D=-0.054$; 95\% Cl: $(-0.163 ; 0.054) ; P$-value $=.32)$. In the random-effects meta-analysis that considered endurance TTE $<100 \%$ of $\mathrm{VO}_{2 \max }$, the $\mathrm{MD}$ of endurance TTE between $\beta 2$-agonists and placebo was not significantly different from zero ( $\mathrm{MD}=1.712 ; 95 \% \mathrm{Cl}$ : $(-1.013 ; 4.437)$; $P$-value $=.21$ ) (Figure 2). (For summary of meta-analysis, sensitivity analysis and evaluation of publication bias, see supporting information).

A fixed-effects meta-analysis was performed using the MD of endurance TTE as an effect measure. No statistically significant difference was found between inhaled $\beta 2$-agonists vs. placebo (MD $=-0.057 ; 95 \%$ Cl: $(-0.165 ; 0.051) ;$-value $=.30)$. However, the meta-analysis considering the effect of oral $\beta 2$-agonists on endurance TTE showed a statistically significant mean increase (MD $=6.029 ; 95 \% \mathrm{Cl}$ ( $2.653 ; 9.385)$; $P$-value $<.001$ ). (For summary of meta-analysis, sensitivity analysis and evaluation of publication bias, see supporting information).

Overall, the results reported here were consistent and showed that inhaled $\beta 2$-agonists do not contribute to the physical performance improvement of healthy athletes. Previous studies have assessed the effect of $\beta 2$-agonists vs. placebo in the changes of $\mathrm{VO}_{2 \text { max }}$, having found that, regardless of the $\beta 2$-agonist, there were no significant effects on physical performance. ${ }^{2,3}$ Moreover, inhaled salbutamol did not show a statistically significant effect on $\mathrm{VO}_{2 \max }$, even when administered at high doses.

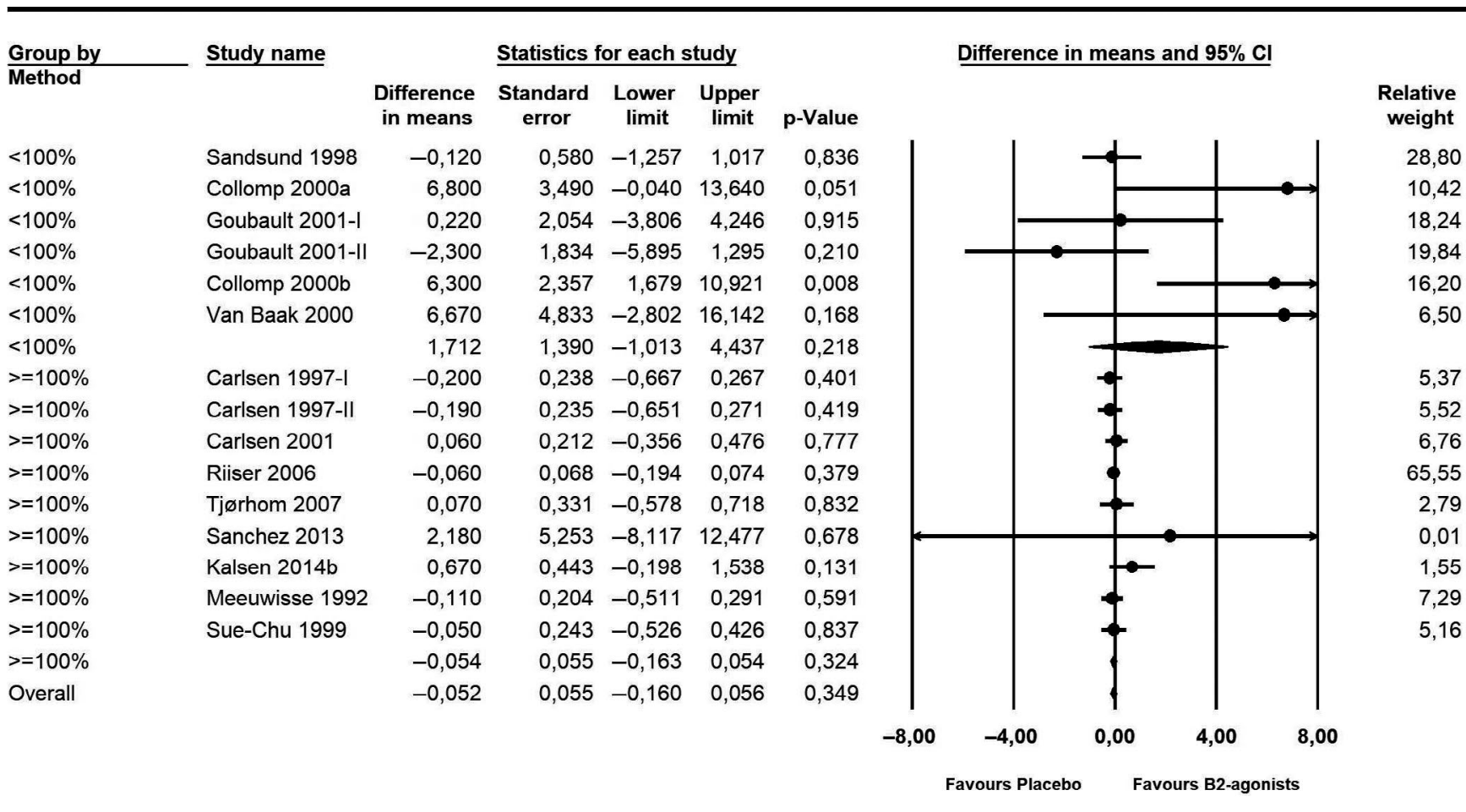

FIGURE 2 Forest plot representing the comparison of MD of endurance time to exhaustion (TTE) between $\beta 2$-agonists and placebo, considering different percentages of $\mathrm{VO}_{2 \max }$ 
When comparing the effect of systemic (oral or IV) salbutamol on $\mathrm{VO}_{2 \text { max }}$, it appears that this $\beta 2$-agonist does not have a statistically significant effect. These results, despite being somewhat inconsistent due to the lack of studies, agree with other studies already performed. ${ }^{4,5}$

Regarding inhaled $\beta 2$-agonists' effect on endurance TTE, no significant effect was shown. However, when considering the oral route, the effect was statistically significant for salbutamol, which explains the prohibition of this route of administration for athletes granted a TUE.

So far, the only systematic reviews and meta-analysis that assessed the effect of $\beta 2$-agonists on physical performance are those described by Kinderman ${ }^{2}$ and Pluim. ${ }^{3}$ Both reviews are dated and considered only a fraction of the studies analysed in the present systematic review, mainly focusing on competitive athletes.

This systematic review and meta-analysis included all existing articles which assessed the effect of $\beta 2$-agonists on physical performance in nonasthmatic athletes and were published until 2018. However, only a few could be included in the meta-analysis, as the others did not present quantitative data. It is important to highlight that it was not possible to evaluate the individual results of the athletes. Additionally, we should be aware that $\mathrm{VO}_{2 \max }$ measures do not represent a direct measure of physical performance. Nevertheless, it is considered a variable of physical fitness and training status of each individual.

Concluding, the results obtained do not show any effects of this class of drugs on physical performance. Moreover, the exceptions currently allowed by WADA do not include any drug that could constitute a competitive advantage. However, further studies should be conducted to assess the effect of $\beta 2$-agonists on physical performance in athletes with asthma or exercise-induced bronchospasm.

\section{KEYWORDS}

doping, performance, sport, $\beta 2$-agonists

\section{CONFLICTS OF INTEREST}

The authors have no conflict of interest to declare.

\section{FUNDING INFORMATION}

This work is supported by national funding through the Portuguese Foundation for Science and Technology, IP, under projects UID/04045/2020 and UIDB/ 00709/2020.

Ana Cardoso ${ }^{1}$

Beatriz Mónico ${ }^{1}$ Jorge M. R. Gama ${ }^{3}$ iD Henrique P. Neiva ${ }^{4,5}$ iD Daniel A. Marinho ${ }^{4,5}$ iD
Olga Lourenço $\mathrm{O}^{1,2}$

${ }^{1}$ Faculty of Health Sciences, University of Beira Interior, Covilhã, Portugal

${ }^{2}$ CICS - UBI Health Sciences Research Centre, University of Beira Interior, Covilhã, Portugal

${ }^{3}$ Centre of Mathematics and Applications (CMA-UBI), University of Beira Interior, Covilhã, Portugal

${ }^{4}$ Department of Sport Sciences, University of Beira Interior, Covilhã, Portugal

${ }^{5}$ Research Center in Sports Sciences, Health Sciences and Human Development, CIDESD, Covilhã, Portugal

Correspondence

Olga Lourenço, CICS - UBI Health Sciences Research Centre, Avenida Infante D. Henrique, 6200-506 Covilhã, Portugal. Email: olga@fcsaude.ubi.pt

\section{ORCID}

Jorge M. R. Gama (iD https://orcid.org/0000-0003-3926-580X

Henrique P. Neiva (iD https://orcid.org/0000-0001-9283-312X

Daniel A. Marinho (iD https://orcid.org/0000-0003-2351-3047

Olga Lourenço iD https://orcid.org/0000-0002-8401-5976

\section{REFERENCES}

1. Allen H, Backhouse SH, Hull JH, Price OJ. Anti-doping policy, therapeutic use exemption and medication use in athletes with asthma: a narrative review and critical appraisal of current regulations. Sports Med. 2019;49(5):659-668.

2. Kindermann W, Meyer T. Inhaled beta2 agonists and performance in competitive athletes. Br J Sports Med. 2006;40(Suppl 1):i43-i47.

3. Pluim BM, de Hon O, Staal JB, et al. beta(2)-Agonists and physical performance: a systematic review and meta-analysis of randomized controlled trials. Sports Med. 2011;41(1):39-57.

4. Violante B, Pellegrino R, Vinay C, Selleri R, Ghinamo G. Failure of aminophylline and salbutamol to improve respiratory muscle function and exercise tolerance in healthy humans. Respiration. 1989;55(4):227-236.

5. McKENZIE DC, Rhodes EC, Stirling DR, et al. Salbutamol and treadmill performance in non-atopic athletes. Med Sci Sports Exerc. 1983;15(6):520-522.

\section{SUPPORTING INFORMATION}

Additional supporting information may be found online in the Supporting Information section. 